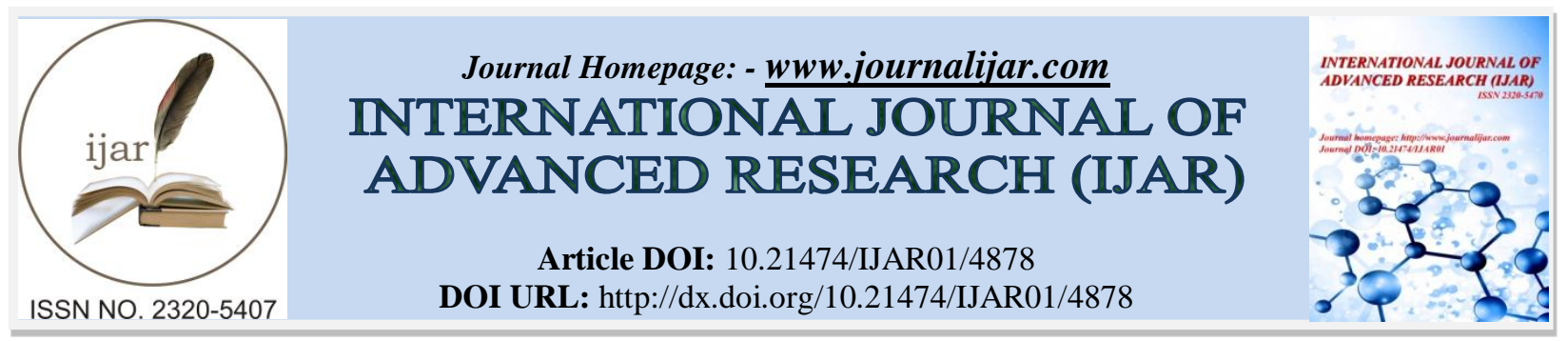

RESEARCH ARTICLE

\title{
A REVIEW ON FACIAL PALSY.
}

Dr. Rahul Tiwari ${ }^{1}$, Dr. Heena Dixit ${ }^{2}$, Dr. Mayank Jangid ${ }^{3}$ and Dr. Pranoti Hiralkar ${ }^{4}$.

1. Post Graduate Student, Department of Oral and Maxillofacial Surgery, Sibar Institute of Dental Sciences, Takkellapadu, Guntur, NTRUHS, Vijayawada, Andhra Pradesh, India.

2. Rungta Institute of Dental Sciences, Bhilai, Pt.RSU University, Raipur, Post Graduate Diploma in Health Care and Hospital Management, Disha College, Raipur.

3. Post Graduate Student, Department of Oral and Maxillofacial Surgery, Vyas Dental College and Hospital, Jodhpur, RUHS, Rajasthan, India

4. Post Graduate Student, Department of Public Health Dentistry, Sibar Institute of Dental Sciences, Takkellapadu, Guntur, NTRUHS, Vijayawada, Andhra Pradesh, India.

\section{Manuscript Info}

\section{Manuscript History}

Received: 18 May 2017

Final Accepted: 20 June 2017

Published: July 2017

Key words:Bells palsy.

\section{Abstract}

Bell's palsy is defined as an idiopathic paresis or paralysis of the facial nerve. The name was ascribed to Sir Charles Bell, who in 1821, demonstrated the separation of the motor and sensory innervation of the face. The incidence ranges from 15 to 40 cases per 100,000, with an equal distribution between the sexes. There is no racial predilection and advancing age may be a risk factor, although this remains controversial. The disease involves the right and left nerves in equal proportions and is recurrent in approximately $10 \%$ of cases it occurs bilaterally less than $1 \%$ of the time. About $8 \%$ of patients report a positive family history of Bell's palsy, with a higher incidence in those with bilateral disease

Copy Right, IJAR, 2017,. All rights reserved.

\section{Introduction:-}

Unlike all other nerve lesions, injuries of the facial nerve not only cause a paresis of the target muscle but also, as the facial nerve is responsible for the range of facial expression, cause serious disturbances in social life. Facial expression being so important in transferring emotion. The facial nerve is a mixed motor and sensory nerve with the main function of innervation of the muscles of voluntary facial expression. Unilateral paralysis permits the unopposed muscles of the unaffected side of the face to exert an abnormal pull on the palsied side. The voluntary or emotional elevation or wrinkling of the forehead, the contraction of the lids and nasal muscles, and the equation of the active side of the month in a smile or grimace contrast with the facial flaccid paralyzed side, the wide staring palpebral tissue with exposed sclera, and the twist of the nose to the unaffected side, following the distorted oral fissure. Drooling and ballooning of the check while eating, persistent epiphora and muffled or garbled speech, are merely outward signs of the mental distress which the patient is suffering.

\section{History:-}

In 1814 Charles Bell sectioned the facial nerve of a monkey, noted that a facial paralysis on the same side resulted, and thus provided that the nerve was responsible for facial expression. Although Herbert Mayo in 1822 offered the first clear description of the function of the facial nerve, Bellinghieri in 1818 anticipated Bell's demonstration of 1821 which conclusively proved that the trigeminal was sensory and that the facial nerve was the motor nerve of the 
superficial muscles of facial expression. Scientific neurology started with Bell. His classic clinical description of facial paralysis in humans is as follows: "The muscles of the cheek on the left side are wastedand there appears to remain nothering but the thin integument, which hangs upon the side of the face, as if dead, without having any action in them or wrinkles, as in the right cheek ; and when he speaks, this cheek is alternately puffed out and then collapsed. The air first distending it as if it were a bag and then escaping at the angles of the mouth. His whole mouth is drawn to the right side, thus producing most remarkable distortion of the face. Whatever action there is in the mouth is altogether owing to the contraction of the muscles on the right side of it ; and left angle hangs loose and it is quite passive ; and the saliva is allowed to flow constantly out of the lower lip on this side". Herbert Mayo 1822 offered first clear description of the function of the facial nerve. Bellinghieri in 1818 anticipated Bell's demonstration of 1821 which conclusively proved that the trigeminal was sensory and that the facial nerve was the motor nerve of the superficial muscles of the facial expression.

\section{Anatomy:-}

The intracranial facial nerve: The supranuclear and infranuclear pathways form the trunk of the facial nerve, which leaves the pons, crosses the subarachnoid space to the internal auditory meatus, and enters the facial canal of the temporal bone. The greater superficial petrosal nerve (the most proximal branch of the facial nerve) arises at the level of the geniculate ganglion. It supplies secretomotor fibres to the lacrimal gland and conveys taste from the soft palate and probably the deep pressure sense and pain from the skin, muscles, and bones of the face. The nerve to the stapedius arises above the chorda tympani in the vertical portion of the facial nerve. It exerts a protective dampening effect upon sound vibrations reaching the iner ear and is activated reflexly by sounds of fairly high intensity. Of the three branches of the nerve within the temporal bones, the chorda tympani is the most peripheral. It arises 4 to $6 \mathrm{~mm}$ above the stylomastoid foramen and contains secretomotor fibers tot eh submaxillary and sublingual glands, as well as taste fibers from the anterior two-thirds of the upper lateral half of the tongue. In connection with the facial nerve after it leave the skull there are two questions to be considered: the relation of the nerve to the parotid gland and the anatomical and functional distribution of the nerve on the face. Relation to parotid gland: As the facial nerve emerges through the stylomastoid foramen, typically situated slightly posterolateral to the styloid process and hence between this and the mastoid process, it is almost immediately comes into relation to the deeper and posterior aspect of the parotid gland. The nerve enters the substant of the parotid gland and divides into two chief divisions an upper temporofacial and a lower cervicofacial one. McWhorter described the two divisions of the facial nerve as passing between superficial and deep part of the parotid gland; on either side of isthmus which unites these two parts; according to his description the superficial and deep parts of the gland thus form lobes which are usually distinct and readily separable, and have a separate duct system, except where are they are united through isthmus. He found that each isthmus had ducts connecting the deeper and superficial parts. Multiple isthmi passing between upper and lower divisions of the facial nerve, but there were multiple isthmi passing between and among the further branches of the facial nerve as it forms the parotid plexus within the gland. The parotid gland, located anterior to and below the lower part of ear, extends subcutaneously muscle and forward over the masseter muscle, and also deeply behind the ramus of the mandible to lie between this and the external auditory meatus and mastoid process. Superficial and deep parts of the gland have been described and regarded both as broadly continuous with each other and as being united only by an isthmus about which rum the branches of the facial nerve; the relationship of the gland to the facial nerve constitutes the chief hazard of the surgery of this gland. Pes anserinus: Within the substance of gland the facial nerve branches into the temporofacial and cervicofacial trunk, just behind the retromandibular vein. In $90 \%$ cases, the two trunks lie superficial to the vein. The trunks branch further to form a perotid plexus (per anaserinus) which exhibits variations in branching pattern. Five main terminal branches arise from the plexus and diverge within the gland. The leave the gland by its anteriormedial surface, medial to its anterior margin and supply the muscles of facial expression.

\section{Branches of Facial Nerve:-}

As the facial nerve emerges from the stylomastoid foramen its first branch is the post aurichlor which runs upward between the parotid gland and anterior border of the sternocleidomastoid muscle and then in the notch between external auditory meatus and the mastoid process; this branch supplies the more posterior muscles of the facial group : the occipitalis, the auricularis posterior and the intrinsic muscles of the auricle. After entering the parotid gland and separating into temporofacial and cervicofacial division it branches further, these branches then usually divide and anastomose to form a plexus a variable number of branches emerge, leave the parotid gland and are distributed to the face. Facial nerve in the face as being divided into five sets - temporal, zygomatic or infraorbita, buccal, marginal mandibular and cervical. The temporal, zygomatic branches of distribution arise from the temperofacial division. The marginal mandibular and cervical branches from the cervicofacial division; however, 
even the two major divisions of the facial nerve may anastomosic each other within the parotid gland; or may anastomose further forward on the face so that both contribute to the buccal branches. It is paralysis of the buccal and marginal mandibular branches which produces the most facial deformity since they are concerned with the highly mobile lips. McCormack, Caldwell and Anson (1945) Classified facial nerves from a hundred dissections, divided them into eight major types with many variants according to the type of branching and major anastomoses. The temporal branches innervate the anterior auricular and post of the superior auricular muscles and the muscles of the forehead, including the major portion of the orbiculoris oculi muscles. The zygomatic branches also supply the orbicularis oculi muscle, the muscles around the nares and elevator of the upper lip; the buccal branches supply most of the musculature around the lip. The lower branches of the buccal and of the marginalis mandibularis supply the musculature of the lower lip and the cervical branches innervate the platysma. The vascular supply: The blood supply of the extratemporal segment of the facial nerve at the stylomastoid foramen is furnished by the branch from the stylomastoid artery (from the posterior auricular artery). The facial nerve also receives twigs from the occipital artery and in the parotid from the superficial temporal and the transverse facial arteries. Fine and delicate arteriae comitantes accompany and enter the terminal branches that leave the parotid gland. As in all nerves, the blood supply of the facial nerve travels along the sheath and dissection must of necessity disturb the delicate branches.

\section{Etiology:-}

The etiology of facial paralysis of varied. The literature of "Bell's palsy" from the early nineteenth century to the present is replete with many causes and a multitude of treatment. The simplest chill, cold or ague or most of the complicated sequence of allergic and immunologic phenomena may herald its onset. Infections : Viral, bacterial or spirochetal cerebrites facial nuclear and endotemporal irritation meningitis, osteomysititis, parotitis have been considered to be causes. The lesions may be due to degenerative changes in the arteries, in the nerves, as in alcoholic neuritis and polynuritis and in the muscles. Developmental anomalies such as central nuclear agenesis, ischemic changes in the pons or fallopian canal, and tumors - intracerebral, intratemporal and parotid - have caused paralysis. Trauma surgical and accidental, due to blast or electricity to scalpel, retractor or a blunt object, has been implicated. For many years surgical incision and neoplastic changes predominated as causal factor. Automobile accidents and cerebral arteriosclerotic disease currently appear to predominate. Any operative procedure distal to the stylomestoid foramen present the risk of severance of the nerve. An incision to explore the F-Z junction in maxillofacial fracture can sever the zygomatic branches. The application of obstetrical forceps can crush the nerve during the delivery. A patient presenting with facial paralysis is a diagnostic challenge. Facial paralysis usually represents manifestation of any number of disorders or abnormalities. Like trauma, neurologic, metabolic, neoplastic, toxic, iatrogenic, idiopathic disorders and infection. The various etiologic factors involved may be broadly classified into three major groups: A) Central (or) intracranial region: Vascular abnormalities, CNS degenerative diseases, Tumor of the intracranial cavity, Trauma to the brain, Congenital abnormalities and agenesis. B) Temporal bone region, Bacterial and viral infections, Cholestratoma, Trauma, Longitudinal and horizontal fractures of temporal bone, Gunshot wound, Tumors invading the middle ear, mastoid region and facial nerve, Iatrogenic causes (surgical injury). C) Parotid gland region: Malignant tumors of parotid gland, Trauma (lacerations or gunshot wounds), Iatrogenic factors, Primary tumours of the facial nerve, Malignant tumor of the ascending ramus of mandible, the pterygoid region and the skin.

\section{The Effects of Nerve Injury:-}

Nerve injuries can be subdivided into various types: Simple block of axon with no histologically detectable changes, destruction of the axon, discontinuity of the sheath, funicular disorganization, and severance of the nerve trunk. Various lesions can be present, depending upon the etiologic factors, whether infection, thrombosis, pressure, direct trauma or impact. The effects of these injuries are degeneration both distally, proximally and the formation and subsequent shrinkage of Schwann cell tubes. Regenerating axons have varying chances of being confiding to their original endoneural tubes and directed to the exact original end organs with out cross-shunting. Inter funicular fibrosis and scar tissue may block the regenerating axons and these are no longer confined to endoneural tubes within the original funiculi. Sunderland (1952) started "the distal stumps retains the capacity of transmitting axones to the periphery for at least twelve months and muscle function can be fully restored following reinnervation even at that time". Recent studies have shown that even several years after the onset of total facial paralysis reinnervation can be resistor. Even mild stretching can case damage to epineural vessels with resulting patchy ischemia. Sever stretching can cause perineural rupture Herniation, followed by endoneuralral fibrosis and impairment of regeneration of regeneration of a grossly intact nerve trunk. Nerve injuries can be subdivided into various types : simple block of the axon with no histologically detectable changes, destruction of the axon, discontinuity of the sheath, funicular disorganization, and severance of the nerve trunk. Various lesions can be present, depending upon 
the etiologic factors, whether infection, thrombosis, pressure, direct trauma, or impact. Traumatic nerve paralysis can be divided into three types: 1 . Neuropraxia, in which only the myelin sheath is affected. It occurs some days after trauma and disappears spontaneously and completely. 2. Axonotmesis, in which the neural element is separated and damaged but the neurilemmal sheath is not interrupted, and the patient may make a spontaneous, if incomplete, recovery. 3. Neurotmesis, in which the neural elements as well as the nerve sheaths are completely interrupted, by either operative intervention or external trauma. Wallerian degeneration occurs with axonotmesis and neurotmesis but not with neurapraxia. If facial nerve paralysis is noted immediately after a laceration, exploration to determine the exact type of injury is indicated. The longer exploration is delayed, the poorer will be the results of repair. Some fibrosis of paralysed facial muscles occurs in a year; after this period the degenerative muscular changes are more progressive, often militating against a satisfactory functional result from any type of nerve repair.

\section{Diagnosis:-}

Clinical examination: Bell's description of a patient with unilateral facial paralysis cannot be improved upon. The patient should be examined in action. The motor function is tested by having the patient wrinkle his forehead, close his eyes, show his teeth, purse his lips, and dilate and contract his nares. As a matter of routine, the face can be subdivided into six examining areas and the response to attempted corrugting, squeezing the eyelids tightly, nose wrinkling, teeth showing, lipspuckering, and in the neck, platysma tightening. Ballooning or puffing out the cheeks, chewing, swallowing, spitting, and speech should also be checked. Subjective sensory disturbances of taste (ageusia) and hering (dysacusis), as well as any unusual history of twitching or facial pain, should be noted. All patients with facial and head injuries or tumors should be routinely checked for possible weakness of the facial muscles. Muscular tone and symmetry are noted at rest and in motion, when the patient smiles or cries spontaneously or talks with great emotion. These maneuvers may point to "thalamic" facial weakness of the supranuclear type, in which voluntary action may be normal but muscular weakness is present during emotional episodes. With the patient's face at rest, changes of the "irritative" type, twitches or even spasms, are sought as evidence of partially returning, or even misdirected, nerve fibrils. The lines of expression usually have disappeared, including the nasolabial fold. As a result of paralysis of the buccinator and the orbicularis oris muscles, whistling or puffing up the affected side. Similarly, the patient drools food and saliva during mastication. There is usually a slight speech defect, and the articulation of labials is interfered with. Paralysis of the platysms can be checked when the patient depresses his chin against finger resistance. Late physical signs include nasal and septal deviation to the contralateral side and, not infrequently, nasal obstruction. Buccal and mucosal protrusion and erosion are prominent in neglected cases. Bell's signs are Abrupt loss of muscular control on one side of the face. Imparting a rigid mask like appearance and resulting in the inability to smile, to close the eye to wink or to raise the eyebrow. The corner of the mouth usually droops, causing saliva to drool on to the skin. Speech becomes slurred and taste may be abnormal. Because the eyelid cannot close, conjunctival dryness or ulceration may occur. Middle aged group people are affected more frequent. (Women > men). Bells phenomenon: Facial paralysis affecting the orbicularis oculi muscle results in an inability to close the eyelids, a condition referred to as lagophthalmos. Because of the loss of orbicularis muscle tone, gravity pulls the atonic lower lid into a sagging position (ectropion). When the lower lid margin and lacrimal punctum are no longer in apposition with the glove, normal tear flow and the lacrimal drainage system are disturbed. Constant exposure of the cornea results in evaporation of the tear film, dryness of the cornea and exposure keratitis. This condition may progress to infection, corneal ulceration and eventual blindness. Fortunately, because of Bell's phenomenon, the eyeball rotates upward and the cornea comes to lie under the upper lid, helping to protect if from trauma and desiccation. However, in patients with a totally paralyzed orbicularis oculi this is usually insufficient to protect the eye. Ocular Signs: In complete unilateral facial palsy the palpebral fissure cannot be closed on the affected side. Although passive closure may occur during sleep because of relaxation of the levator palpebrae superioris muscle, and attempt at closing the eyeball upward and inward, until the cornea passes under the upper lid. The paralyzed lower lid exposes the sclera. Eversion of the lid margin prevents contact of the lacrimal punctum against the eyeball, interfering with the tear drainage. Palsy of the orbicularis oculi muscle hampers collection of the tears by the lacrimal sac. The punctum becomes stenosed and the exposed caruncle hypertrophies. Increased conjunctival exposure leads to increased lacrimal secretion, and the sagging lid collects the tears in the midportion of the lacrimal lake away from the canthi, thus interfering with normal drainage. Location of the lesion: Bilateral facial paralysis is rare. It results in expressionless features, with the inability to smile, to close the eyes, to elevate the eyebrows, to wrinkle the forehead, or to show the teeth. There is profuse tearing and the acts of mastication and speech are considerably interfered with. Bilateral congenital facial paralysis associated with bilateral abducens cranial nerve palsy (Mobius syndrome), although uncommon, is striking and variable in extent and degree and may involve all of the extent and degree and may involve all of the cranial nerves. A distinguishing feature of supranuclear paralysis is the preservation of the action of the orbicularis oculi and frontalis muscles associated with 
paralysis of the lower facial muscles on the side opposite the cerebral lesion. The eye can be closed, the eyebrow elevated, and he forehead wrinkled, but the muscles of the lower two-thirds of the face are flaccid. An unusual facial weakness of the supranuclear type is sometimes detected only when the patient smiles spontaneously and a "thalamic" facial weakness is present. Associated lesions, such as abducens palsy, may accompany facial nuclear lesions secondary to poliomyelitis. Associated nerve palsies and anesthesia of the other cranial nerves must be searched for and noted, especially after trauma and after removal of intracranial tumors. Diagnosis of the site of intratemporal interruption of the nerve. To determine the level of nerve block, the function of the three major branches of the nerve which arise within the temporal bone may be tested. The chorda tympani contains secretomotor fibers to the submaxillary and sublingual glands, as well as taste fibers from the anterior two-thirds of the upper lateral half of the tongue. The gustatory function is tested either by galvanic current, nothing the difference between the metallic taste on the sound side of the tongue and the sensation of the electric shock on the affected side, or by an applicator moistened with a bitter solution. Patients questioned about their reaction to loud sound may give a clear-cut history that the sound of high-pitched children's voices and of the clashing of dishes is almost intolerable in the early stages of paralysis involving the nerve to the stapedius muscle. The greater superficial petrosal nerve (the most proximal branch) arises at the level of the geniculate ganglion. It supplies secretomotor fibers to the lacrimal gland and conveys taste from the soft palate and deep propriceptive fibers. The rate of secretion of the lacrimal gland is studied by Cawthorne's (1956) modification of Schirmer's test: a strip of filter paper hooked over the lower lid acts as a wick; the patient is given a whiff of ammonia, and the rate of flow along it is compared with that of a similar strip applied to the opposite eye. Taste sensitivity of the soft palate may be investigated as described for the tongue. Diagnosis in acute trauma: Facial lacerations producing injury to the facial nerve are more frequent than is generally supposed. Segmental divisions of the facial nerve, anastomoses between the branches of the nerve, and the power of regeneration of the facial nerve (with the exception of the frontalis and marginal mandibular branches) have minimized the number of paralyses after severance. The current increase in the number of maxillofacial injuries, particularly in automobile accidents, has led to an increasing incidence of facial nerve serverance. Facial paralysis may be easily missed in an unconscious patient or in a patient with a bandaged head; unless the paralysis has been specifically looked for initially and the state of facial movements reported in the notes, a history of onset one or two days later cannot be relied upon. Later swelling of the face may further delay diagnosis.

\section{Electrodiagnostic Tests:-}

Electrodiagnostic testing consists of stimulating tests, recording tests, and combined stimulating and recording tests. Stimulating tests: Galvanic tongue stimulation: Anodal galvanic stimulation of the tongue (electrogustometry ) has been developed and consists of measuring the threshold of the anterolateral tongue region to acid taste with the application of a low galvanic current up to 100 microamps from a battery generator. The test is considered normal if the unilateral threshold is less than 10 microamps or there is a difference of 5 microamps between the two sides (Taverner and coworkers, 1967). The patients with abnormal galvanic thresholds do not always develop denervation. In addition, but some patients with normal results have shown later denervation, but if there is a difference of approximately 100 microamps after five days of paralysis, denervation can be expected. This technique however, has limitations, depending upon patient cooperation, and can be abnormal in heavy smokers. Consequently, it is not a definitive answer but may be of value earlier than nerve conduction tests. Chronaxie: Measuring chronaxie is the easiest of the stimulating tests. A chronaximeter is a muscle stimulator so constructed that the strength of electrical stimulus and its duration may be varied through wide limits, and these parameters are read directly from the recording dials on the machine. There are two major types of chronaximeters: the constant cyrrebt type in which the voltage is the variable, and the constant voltage type in which the current flow is the variable. Bipolar electrodes are placed over the motor point of a given muscle. The chronaximeter is set at a given strength of stimulus, long enough to be considered infinite in time. If the strength is sufficient to be threshold for that particular muscle, a resulting muscle twitch is seen. If the strength is not of sufficient intensity, no response cocurs and the intensity must be increased until threshold is reached. Essentially, the test is performed to determine whether the nerve pathway to the muscle is intact. If the nerve muscle pathway is intact, the chronaxie is one millisecond or less. If, however, the nerve is injured, it will be necessary to increase the duration of stimulus at the motor point to a much longer period, so that the muscle fiber is stimulated. Since muscle is not nearly as sensitive to electrical stimuli as intact nerve tissue, it will take a much longer duration of stimulus to fire the muscle and produce a muscle twitch. In other words, the chronaxie in denervation will be prolonged above one millisecond, and one probably can then make a diagnosis of lower motor neuron disease and suggest other tests. Any pathologic process which impairs the ability of the lower motor neuron to conduct an impulse will produce an abnormal chronaxie. Nerve conduction test: The facial nerve is maximally stimulated at the angle of the jaw with surface or concentric needle electrodes in the 
frontalis or orbicularis oris muscles. The evoked muscle potential is displayed on an oscilloscope, and the distal latency is measured from the onset of stimulus. A latency of less than $3.8 \mathrm{msecis}$ considered normal. Studies of normal and abnormal latencies have been done in Bell's palsy (langworth and Taverner, 1963). After seven days, lack of conductibility is indicative of complete denervation and delay of latency compatible with partial denervation. Therefore, conduction latencies at this point in time can provide valuable information in terms of prognosis and treatment.

Recording tests: Electormyography: electromyography is a technique of recording electrical muscle potentials without stimulation. The usual method is to insert a needle electrode into the muscle to be tested, amplify the electropotentials generated by the muscle, visualize these by means of a cathode ray oscilloscope, and make them audible through a speaker. The procedure for electromyography is fairly simple. The needle electrode, which can be monopolar, bipolar, or multipolar, is thrust into the muscle. The muscle is tested at rest and on slight voluntary contraction. Amplification of about one million times is achieved as the amplitudes of these small potentials are in the millivolt range. The cathode ray oscilloscope indicates potentials by means of a beam of electrons, and the horizontal sweep of the electron beam across the oscilloscope is calibrated to give the duration of the potentials generated. The vertical plates after suitable amplification show, by their deflection of the beam, the amplitude and the shape of the potentials generated. The impulses form the cathode ray tube may be photographed with either a still or movie camera. Effects of upper motor neuron lesion and lower motor neuron lesion:

\begin{tabular}{|c|c|c|c|}
\hline \multicolumn{2}{|r|}{ Effects } & Upper motor neuron lesion & Lower motor neuron lesion \\
\hline \multirow{7}{*}{$\begin{array}{l}\overline{\mathscr{J}} \\
. \\
.\end{array}$} & 1. Muscle tone & Hypertonia & Hypotonia \\
\hline & 2. Paralysis & Spastic type of paralysis & Flaccid type of paralysis \\
\hline & 3. Wastage of muscle & No wastage of muscle & Wastage of muscle occurs \\
\hline & 4. Superficial reflexes & Lost & Lost \\
\hline & 5. Plantar reflex & Abnormal plantar reflex-Barbinski’s sign & Plantar reflex-absent \\
\hline & 6. Deep reflexes & Exaggerated & Lost \\
\hline & 7. Clonus & Present & Lost \\
\hline \multirow{3}{*}{ 节 } & 8. Electrical activity & Normal & Absent \\
\hline & 9. Muscles affected & Groups of muscles are affected & Individual muscles are affected \\
\hline & 10. Fascicular twitch in E.M.G. & Absent & Present \\
\hline
\end{tabular}

Upper motor neuron and lower motor neurons: The neurons of the motor system are divided into upper motor neurons and lower motor neurons depending upon their location and termination.

Upper motor neuron: Upper motor neurons are the neurons in the higher centers of brain, which control the lower motor neurons. There are three types of upper motor neurons, which are: Motor neurons in the cerebral cortex. The fibers of these neurons form corticospinal (pyramidal) and corticobulbar tracts. Neurons in the basal ganglia and nuclei in brainstem. Neurons in the cerebellum. The motor neurons in the cerebral cortex which give origin to pyramidal tracts belong to the pyramidal system and the remaining motor neurons belong to extrapyramidal system. Some controversy exists in including the neurons of extrapyramidal system under the category of upper motor neurons. However, considering in terms of the definition, the neurons other than the lower motor neurons are to be named as upper motor neurons. Lower motor neurons: Lower motor neurons are the anterior gray horn cells in the spinal cord and the motor neurons of the cranial nerve nuclei situated in brainstem, which innervate the muscles directly. Thus, the lower motor neurons constitute the "final common pathway" of motor system. The lower motor neurons are under the influence of the upper motor neurons. Applied physiology-effect of lesions of motor neuron: The effects of lesions of upper motor neurons and lower motor neurons. The effects of lower motor neuron lesion are the loss of muscle tone and flaccid paralysis. The effects of upper motor neuron lesion depend upon the type of neuron involved. The following are the effects of upper motor neuron lesion: The lesion in pyramidal system causes increase in the muscle tone - hypertonia and spastic paralysis. Spastic paralysis involves only one group of muscles. Lesion in basal ganglia produces hypertonia and rigidity involving both flexor and extensor muscles. Lesion in cerebellum causes decrease in muscle tone-hypotonia, muscular weakness and in coordination of movements.

\section{Conservative / Medical Management:-}

Treatment of facial palsy: Statistical degress of benefit from drugs treatment - Prednisone + acyclovir $>$ prednisone $>$ acyclovir. More benefit when $t / t$ started within 3 days of onset. No benefit if $t / t$ is started more than 10 days after onset. Corticosteroids: Use within a week of onset. Adult: prednisone $80 \mathrm{mg}$ qd x 5 days. Children: prednisone $1 \mathrm{mg} / \mathrm{kg} /$ days. After initial dose: Taper off over 7 to 10 days. Acyclovir: Use within 3 days of onset. Adults- 2,000 $\mathrm{mg}$ per day (400 mg $5 \mathrm{x} /$ day) for 7 days. With Varicella zoster 4,000 mg per day. Children: $80 \mathrm{mg} / \mathrm{kg}$ per day for 5 
days. More effective in Ramsay-Hunt syndrome. Alternative antiviral: valacyclovir. Protect eye from exposure. Eye drops. Facial exercise and massage. Analgesics such as aspirin, acetaminophen or ibuprofen, may relieve pain.

\section{Reconstruction in Facial Palsy:-}

The reconstructive techniques used are: Static Suspension by: a) Fascia b) Dermis c) Silastic rubber. Stabilization by: a) Dermal flaps b) Marlex c) Bone fixation. Dynami: Muscle transfer a) Temporalis b) Masseter c) Digastric d) Sternocleidomastoid e) Muscle grafts (Free). Inorganic motors: a) Metal spring (upper lid) b) Silastic bands - lip and lid c) Lid weights d) Lid magnets Sphincteric reconstruction Orbital: a) Canthoplasties: Oral b) Bucal-oral reconstruction. Nares. c) Septal corrective surgery. Control of antogonist muscle a) Neurectomy Temporary chemical, Permanent- surgical b) Myomectomy. Face-lift operations a) Modified cheek plasty b) Blepharoplasty c) Nasolabial excision d) Supraorbital excision and brow lift e) Temporal lift f) Labial and mucosal excisions. Surgical formation of Folds and wrinkles a) Construction of nasolabial fold b) Forehead furrows. Mechanical aids a) Toothpick in the mouth. b) Tape c) Pipe. Muscle Transfers: Muscle transfer entails the transposition of the whole, or a portion, of the belly of the muscle with an adequate blood and nerve supply. The insertion or the origin of the transplant is transferred. The transposition of muscles innervated by the trigeminal nerve is by far the most popular of the muscle transpostion techniques. In transferring muscle, the static deformity must be corrected, the muscle must not be overstretched, and its line of pull must be sufficiently long in a relatively straight direction to function. Muscle transfer was first used in facial paralysis by Lexer (1908), when the anterior half of the masseter muscle was split and transferred to the muscles of the upper and lower lips. Temporalis strips similarly attached to the upper and lower eyelids were also used. Massager muscle transfer combined with fascial suspension. Technique: Exposure of the lower border of the mandible is made through a submandibular incision. The assistant's hand on the cheek retract the lax submaxillary skin superiorly so that the scar will lie under the mandible. The skin incision continues directly to the lower border of the mandible to expose the insertion of the masseter muscle. The superficial surface of the masseteris cleared, the facial covering is elevated from the anterior half, and the branches of the anterior facial vein and external maxillary artery are ligated. The periosteum of the mandible is incised and freed along the inferior border for approximately two-thirds the length of the insertion. Subperiosteal elevation of both the superficial and deep portions of the masseter from the mandibular ramus with an elevator permits elevation and detachment of the anterior two-thirds by splitting the muscle fibers and incising the subjacent periosteum for about two thirds of its vertical length. A dry surgical field will often permit identification of the motor nerve entering posteriorly and branching in the upper third. A careful posterior separation, parallel to the muscle fibers, should be all that is required. The split should be no more than $4 \mathrm{~cm}$ from the inferior border. If more length is required, the entire masseter should be freed and advanced forward. A nasolabial fold incision provides access for careful elevation of the skin over the superficial muscles around the angle of the mouth and lips, and permits the removal of redundant skin. Broad exposure of the superficial muscles allows attachment of the wide divided end of the masseter muscle flap to the underlying orbicularis oris muscle by with Dacron sutures. The position of the angle of the mouth is overcorrected both laterally and superirorly. In closing, multiple 5-0 white Dacron sutures attach the masseter muscle to the dermis at the nasolabial fold to mimic the musculodermal insertion and thus accentuate the fold. Temporalis Muscles Transfer: Facial Transfer. Temporalis muscle transfer to the eyelids, the ala of the nose, the upper and lower lips, and the commissure of the mouth is a dynamic, controlled reconstruction with bony fixation and muscular and dermal attachments which can be secondarily readjusted. Technique. Through and extended facelift incision, the temporalis fascial is exposed and raised superiorly along with the pericranium and inferiorly as far as the zygomatic arch. A quadrilateral fan-shaped flap approximately 8 to $10 \mathrm{~cm}$ in width is outlined, and the upper incision is made $1 \mathrm{~cm}$ above the junction of the temporalis fascia and the pericranium. The lateral incision through the fascia is extends inferiorly almost to the zygomatic arch, becoming narrow as the lines of muscle fibers also narrow, and is deepened bluntly though the fibers to the temporalis fossa to avoid cutting the deep temporal nerves which are branches of the anterior division of the mandibular nerve (fifth cranial nerve). The pericranial-muscleaponeurosis mass is dissecte until it rolls down freely. Dissection with a special periosteal elevator allows the ranches of the nerve to be felt rather than seen; denervation must be avoided. The dissected muscle complex falls over the zygomatic arch. The aponeurosis is increased over the arch and is elevated from the muscle mass. Should the pericranial-aponeurotic attachments seem tenous, reinforcing mattres sutures are inserted into each of the major segments of the muscle belly: the anterior third, to restore the orbital sphincter; an inferior slip of aponeurosis to the ala of the nose (the nasal sphincter); and the posterior two thirds, to restore the oral sphinter. Temporalis muscle tendon and coroniod process transfer: Through an intraoral incision, McLaughlin (1925) detached the coronoid process and, by means of fascial strips, attached the temporalis muscle insertion to the angle of the mouth. 


\section{Inorganic Implants:-}

Static methods to relieve distortion employ the suspension of paralyzed facial muscles by various materials, varying form aluminum, bronze, wire, and silk to stainless steel and tantalum. There are advocates for the use of nonreactive plastic tapes of polyethylene, Teflon, or Sialastic with or without either Marlex of Dacron mesh reinforcement and/or bonded polyurethane foam for organizational tissue attachments. Early results with these materials may prove to be excellent. Experience over a period of 30 years has led to a distrust of inorganic material placed in a position of suspension or tension in superficial locations. The reviews of Backdahl and D Alesion (1958) and embarks on the ase of inorganic materials for facial suspension. Transplants of Dermis: Strips of dermis of width and length comparable to that of strips of fascia inserted in the same manner have a tendency to stretch more and make it more difficult to predict the amount of postoperative stretching. Dermis and lyophilized fascia are more liable to stimulate fibrous proliferation than autogenosu fascia, for they fragment and disintegrate early. Postoperative subcutaneous infections are higher with dermal implants than with fascia, because saprophytic infection carried into the repaired field is manifested many days or weeks after the operation rather than in the usual 12 to 36 hours after operation (pick, 1949).Nonetheless, dermis can be and is still used in an occasional patient. Fascial Transplants: The use of autogenous fascia for blepharoptosis was initiated by payr an applied to the treatment of facial paralysis by Stein (1913). However, it was Blair's advocacy (1926) of the suspension of paralyzed facial muscles by fascia lata bands that popularized the method. STAGE I - Circumoral Fascial Lata Band: If the paralysis is of excessively long standing, a strip of fascia 0.5 to $0.75 \mathrm{~cm}$ wide is introduced into the orbicularis oris muscle or its remnants to circumscribe the mouth through four small external incisions, one at each of the angles and slightly to the side of the midline, and one each for the upper and lower lips. The strip is threaded through a blunt fascia needle carrier or scissors which are perforated near the tip of the blades. The fascial strip is introduced through the tissues of the lip, form the unaffected side around the mouth, as shown in A, coming out again on the unaffected contralateral side. The free ends are crossed and the lips manipulated to "a position of optimal symmetry", to be fixed, there with interrupted silk sutures symmetry", to be fixed there with interrupted silk sutures joining the oribicularis muscle to the fascial loop. The loop is closed by a knot which is secured by a suture. Postoperatively, double-tailed collodion gauze splints are placed, one on each side of the mouth, each overlapping the other, to maintain a buttonhole splint dressing for ten days to two weeks, Fascia-muscle healing is not complete until after five weeks, and a second stage should be deferred until that time.

\section{Facial Nerve Grafts:-}

Preoperative considerations: when resection of the facial nerve may be necessary in the course of surgery, whether intracranial, intratemporal, or extratemporal, a careful evaluation of the probability of nerve resection should be made, and a detailed plan for either immediate or staged replacement of the nerve should be outlined. Repair of the facial nerve can be accomplished by short-circuiting grafts. Restitution of the continuity of the facial nerve between its origin from the pons to the facial musculature is fesible. Approach: Elective exposure is by a preauricular incision around the ear lobe and across the mastoid, curving downward across the sternolceidomastoid muscle in a transverse crease. After the flaps are raised, a cleavage plane is found between the cartilage of the auditory canal and the mastoid posteriorly, and the parotid capsule, which will allow the posterior parotid margin to be elevated anteriorly. The facial trunk and its enveloping fascia are found under the sternocleidomastoid muscle. The stylomastoid foramen is located above the origin of the posterior belly of the diagastric muscle on the mastoid. The removal of asegment of a long overhanging mastoid process is not difficult and can simplify the exposure. The exposed tympanomastoid fissure can also point to the stylomastoid foramen, which is 6 to $8 \mathrm{~mm}$ beneath its "drop-off" point. Another method is the exposure of a peripheral branch by a routine preauricular parotidectomy incision, elevating the flaps and identifying any of the branches, which can be followed proximally to the trunk. Choice of grafting procedure: Facial nerve repair by using a segment of the sensory cervical plexus, incorporating the greater auricular nerve with the main trunk or branches of the segments of $\mathrm{C} 3$ or $\mathrm{C} 4$, is generally used, although the sural nerve has also served as a donor site. Filaments of the nerve graft have been placed directly into muscle. Without direct nerve approximation, with return of muscle function as a result of this procedure. In the selection of the donor nerve and type of graft, the cross section of the graft, or grafts, should be at least equal to that of the nerve to be replaced. The donor nerve should be constant in position and readily accessible, either through the same incision or through one that does not involve a pressure area the resultant sensory defect should be minimal in quantity and extent. The sural nerve and the external saphenous nerve are advocated by many; the greater auricular or one of the cutaneous branches of the cervical plexus, which are available without requiring an additional operative site. The grafts should lie in a healthy, wellvascularized bed from which all scar tissue should be excised. The alternative of bypassing a scar by using a longer graft rather than passing the nerve through relatively avascular tissue should be considered. There is some shrinkage in a long graft, and a graft at least 15 per cent longer than the defect would ordinarily 
require should be used. When the nerve stumps are resected, as for end-to-end anastomosis, it is advisable to avoid mobilizing the proximal segment extensively, to prevent disturbing the longitudinal branches of the vasculature. It is important to resect the distal neuroma; fibrosis in the distal stump can be responsible for failure of the procedure. Morever, resecting the distal stump does not strip the vasculature and merely entails the use of a longer graft. The avoidance of forcible recross-facial nerve transplantation for the restoration of some expressive and emotional movements. Cross-Face Nerve Transplantation: Smith (1971) attempted "micronerve" transfers from the normal to the palsied side to restore facial control and symmetry. With unilateral facial paralysis of various lengths of time, a nerve autograft, averaging $9 \mathrm{~cm}$ in length, was used to connect the paralyzed muscles to their corresponding "normal" muscles on the opposite side of the face. The buccal and zygomatic nerve branches were rerouted and reconnected by this technique. A bilateral $5 \mathrm{~cm}$ incision paralleling but $2 \mathrm{~cm}$ lateral to the nasolabial fold allowed the identification of a neural plexus (branches of zygomatic and buccal portions) at a point $1.5 \mathrm{~cm}$ below the malr prominence. Stimulation of this plexus on the normal side resulted in contraction of the infraorbital portion of the orbicularis oculi muscle, the small muscles of the nose (procerus and nasalis), and the muscles controlling the corner of the mouth and upper lip (qardratus labii superioris, zygomaticus, and levator anguli oris). On the normal side, nerve branches going to the orbicularis were divided distal to the plexus. On the paralyzed side of the face, one or two nerve branches were divided proximal to the plexus and connected by the sural nerve graft across the upper lip between the two incisions. Anastomoses between the sural graft and nerve branches on each side were made under microsurgical control of 10 to 16 power, with the use of "Skewer" sutures through the nerves, which were remove a week following surgery. Postoperatively, improvement is facial symmetry became evident at three of six months. In all three cases some controllable muscle contraction was gained in the initially paralyzed orbicularis oculi and the zygomaticus, quadratus, and levator muscles.

\section{Complications of Reconstructive Procedure:-}

Complications are those encountered in the use of grafts of fascia or flaps of muscle throughout the body. The grafts may stimulate excessive reaction, contract, pull loose, or become infected. Similarly, the muscle flaps may be overstretched, or their vascular supply may be interrupted by edema, excessive tension, and kinking around a turn or over a bony prominence, or they may be strangulated by a fascial strip. Subcutaneous hematoma should be immediately evacuated, even if it is necessary to open the entire incision in the operating room under anesthesia. Fixation of the tissues by late fibrosis or secondary infection may follow and obliterate facial movements and prevent secondary procedures. Adequate postoperative splinting should prevent the pulling out of fascial bands or muscular attachments if they have been placed under an adequate amount of tension. The most obvious and usual deficiency is inadequate excursion, and probably the commonest cause is adhesion. Because the extension of the muscle is insufficient, the muscle is restricted in range, and full motion cannot be restored; tenolysis should not be considered until after the tendon is well healed and the excursion and reduction instituted. A second cause of failure of motion is loss of continuity due to dehiscence at either the proximal or distal junction. Similarly, after exploration and resuture, a five-week rest should elapse prior to instituting graduated active motion. In patients with partial facial paralysis in whom elastic mesh is used, the splinting mesh may prevent stretching during the period of healing. Yet these may pull out, as have that wire fixations at the distal end of the reconstructed sphincter.

\section{Special Procedures in Facial Paralysis:-}

Surgical Simulation of Wrinkles: Surgical formation of facial wrinkles is achieved by the deliberate placing of an incision so that the healed, and possibly stretched, scar will simulate a natural crease, fold, or furrow, and by fixation of the dermis to the underlying muscle transplants. The purposeful fostering of "scar healing" in incisions placed to represent wrinkles corresponding to those on the nonparalyzed side of the face is another method (Frnackelton, 1952). The incision is allowed to heal spontaneously. Correction of palpebral deformities and the relief of Epiphora: Parapysis of the orbicularis oculi muscle of lagophthamimose involves all three of the portions of the muscle, i.e., the pretarsal, the preseptal, and the orbicular, and its early correction is important. The tight closure of the eye is lost, the lower lid droops away from the globe and becomes everted, and impaired drainage, pooling, and epiphora add to the classic houndlike eye. Kazanjian and Converse (1974) have outlined the variety of reasons for epipohor ain facial paralysis "the paralyzed orbicularis muscle cannot dilate the lacrimal sac; due to the relaxation of the eyelid, the punctum is not in contact with the eye ball and is often stenosed; the caruncel which is exposed by the lagophthalmos becomes hypertrophied; the tear lake lies in the middle of the sagging lid and away from the canthi; increased lacrimal secretion due to exposure. Interference with normal drainage leads to increased loss of tears by evaporation, thus the need for increased activity of the lacrimal gland. Methods are available for both temporary and permanent palsies. A grouping of techniques may be established to include; 1) various medial and lateral canthoplasties 2) passive elevation of the lid by fascial silastic bands or slings or a dermal flap 3) temporalis muscle 
transfer; 4) tensing the flaccid cheek by a face-lifting operation and corrective blepharoplasties; 5) improving drainage by dilatation of the punctum and increasing by dilatation of the punctum of the canaliculus. Lid Magnets Muhlbauer, Segeth and Viesmann (1973) have implanted two miniaturized, siliconized curved magnets beneath the orbicularis muscle near the upper and lower eyelid margins. The tarsal plates prevent eruption of the rods into the conjunctival sacs. The magnetic force employed in the open eye position for the double magent system was 200 to $300 \mathrm{mp}$, and this corresponds to the lost muscle tone of the orbicularis muscle, thus preventing upper lid restraction, corneal irritation and epipohora. Normal lid opening is maintained by the intact levator mechanism. Initally the lid magnets are taped to the lid margins to determine the ideal magnet force. When this is established, the lid magnets are implanted under local anesthesia.

\section{External Mechanical Support for Facial Paralysis:-}

External mechanical aids, while employed by the ancients find little application in the present day. A restraining hook set in the depressed angle of the mouth can be supported from a loop around the ear, by a leather, plastic, or plaster headdress, or a dental attachement. Similar elevators for lids can be attached to eyeglasses. Bands of adhesive strapping, or colloidion strips, presently used postoperatively to splint the facial muscles, have been advocated for lifting the erverted ptotic lower lid, or for restraining the edematous, sagging lip. These temporary measures are inferior to the simple surgical maneuveres are inferior to the simple surgical maneuvers suggested earlier in the text. Appliances are value at times, and mention should be made of the Chicago lawyer who effectively disguised his lower facial paralysis with a tightly clenched pipe jutting out of the angle of his mouth, the female dentist two never relinquished here long cigarette holder; and the East Texas farmer with the matchstick toothpick in his molars for 25 years, obscuring a pronounced facial paralysis.

\section{References:-}

1. Converse J.M. Reconstructive Plastic Surgery. 2ND Edition. W. B. Saunders Co. Philadelphia, London, Toronto; 1977.

2. Eisele, D. W., \& Smith, R. V. (2012). Complications in Head and Neck Surgery. [S.L.], Elsevier.

3. Hupp, J. R., Ellis, E., \& Tucker, M. R. (2008). Contemporary Oral and Maxillofacial Surgery. St. Louis, Mo, Mosby Elsevier.

4. Killey And Kay's Outline Of Oral Surgery. Pt.1, 2ND Ed / Gordon R. Seward, Malcolm Harris, David A. Mcgowan, 1939- Et Al. 1987, Wright, Bristol.

5. Krespi, Y. P., Krespi-Ossoff, \& Ossoff, R. H. (1993). Complications in Head and Neck Surgery. Philadelphia [U.A.], Saunders.

6. Kruger, Gustav $1^{\mathrm{ST}}$ Ed. Textbook of Oral And. Maxillofacial Surgery. 6TH Ed. St. Louis, Mosby, 1984.

7. Little,James W., And Falace, Donald Dental Management of The Medically Compro- Missed Patient. 2D Ed.St. Louis, Mosby, 1984.

8. Okeson, J. P., \& Bell, W. E. (2014). Bell's Oral and Facial Pain. Chicago, Quintessence Publications.

9. Papazian, Martin R. et al. Management of Bell's palsy. Journal Of Oral And Maxillofacial Surgery, Volume 51, Issue 6, $661-665$.

10. Peter Ward Booth, Barry Eppley, Rainer Schmelzeisen. Maxillofacial Trauma and Esthetic Facial Reconstruction. $2^{\mathrm{ND}}$ Edition. Elsevier Saunders Publication, Missouri; 2012.

11. Raymond J. Fonseca and Robert V. Walker. Oral and Maxillofacial Trauma, Wb Saunders, Philadelphia, 1991, $1^{\mathrm{ST}}$ Edition.

12. Richard A. Pertes, Sheldon G. Gross. Clinical Management of Temporomandibular Disorders and Orofacial Pain, Oct 1995.

13. Shafer's. Textbook of Oral Pathology (6TH Edition) (2009) Edited By R. Rajendran, B. Sivapathasundharam.

14. Surgical Repair of Facial Nerve Indian Journal of Oral And Maxillofacoal Surgery Special Issue 1998.

15. Wood,Norman K.,And Goaz,Paui.W. Differential Diagnosis of Orallesions.St.Louis Mos-By, 1985.

16. Worthington, P., \& Evans, J. R. (1994). Controversies in Oral and Maxillofacial Surgery. Philadelphia, Saunders. 